\title{
Lady or Gentleman? Sex Differences in Osseous and Cartilaginous Structures of the Knee
}

\author{
Lauren C Pringle, $M D^{1}$, Miho J Tanaka, $M D^{2}$ and Laura M Fayad, $M D^{3^{*}}$
}

\begin{abstract}
${ }^{1}$ Diagnostic Radiology Resident, The Russell H. Morgan Department of Radiology and Radiological Science, The Johns Hopkins School of Medicine, USA

${ }^{2}$ Assistant Professor, Department of Orthopaedic Surgery, The Johns Hopkins School of Medicine, USA

${ }^{3}$ Professor of Radiology, Orthopaedic Surgery \& Oncology; Chief of Musculoskeletal Imaging; Director of Translational Research for Advanced Imaging; The Russell H. Morgan Department of Radiology and Radiological Science, The Johns Hopkins School of Medicine, USA
\end{abstract}

*Corresponding author: Laura M. Fayad, MD, Professor of Radiology, Orthopaedic Surgery \& Oncology, Chief of Musculoskeletal Imaging, Director of Translational Research for Advanced Imaging, Department of Radiology and Radiological Science, The Johns Hopkins School of Medicine, Lisa Morales, USA, Tel: 443-287-6032

\begin{abstract}
Objective: To identify anatomic sex differences in the osseous and cartilage structures of the knee.

Materials and methods: We performed a comprehensive review of imaging investigations of knee morphology comparing male and female subjects using PUBMED, with search terms for CT/MRI, knee anatomy, and gender. Inclusion criteria were primary imaging investigations of knee osseous/cartilage anatomy (minimum 15 subjects); exclusion criteria were studies of post-operative patients, those with comorbid orthopedic conditions, and review articles. Sex differences in osseous/cartilage anatomy were extracted and aggregated.

Results: Of 1550 citations reviewed, 84 studies met inclusion criteria and fell into 5 categories: Bony axes of the knee $(n=21)$, morphology of the distal femur $(n=38)$, proximal tibia $(n=15)$ and patella $(n=8)$, and articular cartilage ( $n$ $=16$ ). Knee axes studies suggested trochlear groove-tibial tuberosity distance and posterior tibial slope may be greater in females. Distal femur studies showed larger metrics in males, and narrower metrics in females with smaller intercondylar notch volumes. Regarding tibial morphology, females had smaller tibias (normalizing for height), with narrower tibial plateaus and deeper medial tibial plateaus. Female patellas were smaller and different enough in morphology to be accurate in predicting sex in forensic studies. Cartilage studies mostly suggested that articular cartilage thickness and volume were greater in males.
\end{abstract}

Conclusion: Sex differences in anatomic structures of the knee have been reported. Given the increasing emphasis on individualized medicine, the emergence of sex-based diagnosis and treatment protocols, radiologists should be aware of anatomic sex differences.

\section{Introduction}

Women's sports medicine is a growing field in the world, as research has shown that women are more susceptible to certain sports-related injuries and require tailored treatment and prevention programs. An understanding of anatomic sex differences is important in the recognition of sex-related injury patterns. In the knee, most researches have focused on the Anterior Cruciate Ligament (ACL), with orthopedic surgery, physical therapy, and other clinical sports medicine fields producing the majority of the research regarding the functional and biomechanical aspects of knee stability and ligament differences. Women are known to have 2-8 times more frequent non-contact $A C L$ injuries than men. While the etiology and implications for this sex difference remained unclear, functional, hormonal and anatomic factors may play a role, and sex differences in bone anatomic features have been studied, as they relate to the $A C L$

Citation: Pringle LC, Tanaka MJ, Fayad LM (2019) Lady or Gentleman? Sex Differences in Osseous and Cartilaginous Structures of the Knee. Int J Sports Exerc Med 5:153. doi.org/10.23937/24695718/1510153

Accepted: November 09, 2019; Published: November 11, 2019

Copyright: (c) 2019 Pringle LC, et al. This is an open-access article distributed under the terms of the Creative Commons Attribution License, which permits unrestricted use, distribution, and reproduction in any medium, provided the original author and source are credited. 
[1]. For example, sex differences had been suggested in the posterior tibial slope of injured knees, a parameter which had been shown to play a role in knee stability in addition to $A C L$ injury $[2,3]$, and differences in notch dimensions and lim alignment may be a causal factor in the developing of tears of the ACL [1]. However, there has been no systematic comparison of knee anatomy as it related to gender differences.

Another area of research relating to bone and cartilage sex differences has been performed outside the acute sports medicine practice, with the goal of personalizing arthroplasty components. Sex-specific implants are now being used clinically, despite some disagreement on whether the differences are truly related to sex or simply the size of the femur and tibia [4]. Additionally, whether sex-specific components improve outcomes after surgery is a controversial topic on its own [5].

Hence, we sought to evaluate the current literature to identify areas of agreement, controversy, and knowledge gaps that may better inform both radiologists and sports medicine clinicians about sex differences in the anatomy of the knee. If radiologists can accurately identify patients at risk of injury, can detect risk factors for post-operative complications, or help aid the clinical and surgical team in operative planning or rehabilitation planning by noting sex differences, the field of radiology will be invaluable in customizing care for patients moving forward. Thus, the purpose of this study was to perform a comprehensive review of the English literature for original scientific researches on sex differences in osseous and cartilage anatomy of the knee.

\section{Materials and Methods}

\section{Search strategy}

To identify original articles for this review, a computerized MEDLINE database search was performed through the PubMed service of the National Library of Medicine for articles in the English language with the search criteria listed (Figure 1). The search included articles published between 2001 and December 2016. Limiting the search to articles in English was done for practical reasons, but very few articles were eliminated using this filter. A manual search and perusing of reference lists was also performed in MEDLINE following the comprehensive search, to identify any potential additional primary studies. The search was performed on March 24, 2016.

\section{Study selection}

Inclusion criteria were primary investigations of cross-sectional imaging of knee anatomy by Computed Tomography (CT) or Magnetic Resonance Imaging (MRI) in males and females of all ages. We excluded studies with fewer than 15 subjects due to small sample size. Studies examining injury patterns, functional anatomy, anatomy of soft tissues (tendons, ligaments, muscles, menisci), and knee development were not included in this study. We also excluded studies focusing on modalities outside of $\mathrm{CT} / \mathrm{MR}$, such that studies examining differences based on radiography and nuclear medicine modalities were rejected. Studies reviewing joints other than the knee (e.g. the hip), studies in patients with comorbid conditions affecting the knee (example: Arthritis), studies in postsurgical patients and review articles

\footnotetext{
("Magnetic Resonance Imaging"[mh:noexp] OR "magnetic resonance imaging"[all] OR "magnetic resonance imagings"[all] OR "MRI"[all] OR "MR imaging"[all] OR "CT"[tiab] OR "Tomography, X-Ray

Computed"[mh:noexp] OR "Four-Dimensional Computed Tomography"[mh] OR "Tomography, Spiral

Computed"[mh] OR "Multidetector Computed Tomography"[mh] OR "computer assisted tomography"[all] OR

"computed tomographic angiography"[all] OR "computed tomography"[all] OR "electron beam tomography"[all] OR

"computer tomography"[all] OR "optical tomography"[all]) AND

("Knee injury"[all] OR "Knee anatomy"[all] OR "knee joint"[mh] OR "knee"[mh] OR "knee injuries"[mh:noexp] OR "tibial menisci"[all] OR "tibial meniscus"[all] OR "patellofemoral joint"[all] OR "patellofemoral joints"[all] OR "knee dislocation"[mh] OR "knee dislocation"[all] OR "knee dislocation"[all] OR "patellar dislocation"[mh] OR "patellar dislocation"[all] OR "anterior cruciate ligament"[mh] OR "anterior cruciate ligament"[all] OR "ACL"[all] OR "medial collateral ligament"[all] OR "MCL"[all] OR "lateral collateral ligament"[all] OR "LCL"[all] OR "posterior cruciate ligament"[mh] OR "PCL"[all] OR "posterior cruciate ligament"[all] OR "patellar ligament"[mh] OR "patellar ligament"[all] OR "patellofemoral ligament"[all] OR "patellar tendon"[all] OR "medial collateral ligament, knee"[mh] OR "lateral meniscus"[all] OR "medial meniscus"[all] OR "patella"[mh] OR "patella"[all] OR "patellar"[all] OR "tibial plateau"[all] OR "distal femur"[all] OR "intercondylar notch"[all] OR "femoral notch"[all] OR (("femur"[all] OR "femoral"[all] OR "knee"[all]) AND ("medial condyle"[all] OR "lateral condyle"[all] OR "trochlea"[all])) OR "tibial cartilage"[all] OR "femoral cartilage"[all] OR "patellar cartilage"[all] OR "knee cartilage"[all] OR (("meniscal tear"[all] OR "articular cartilage"[all]) AND "Knee"[all]) OR (("Fractures, Bone"[mh] OR "fracture"[all] OR

"fractures"[all] OR "bone bruise"[all] OR "bone contusion"[all] OR "osteochondral defect"[all] OR

"osteochondritisdissecans"[mh] OR "osteochondritisdissecans"[all]) AND ("knee"[all] OR "femur"[all] OR

"femoral"[all] OR "patella"[all] OR "patellar"[all] OR "tibia"[all] OR "tibial"[all])) OR "quadriceps tendon"[all]) AND

(Gender[tw] OR "Sex"[mh] OR "sex"[all] OR ((Male[tiab]OR males[tw] OR men[tw] OR man[tw]) AND (female[tiab] OR females[tw] OR women[tw] OR woman[tw])) OR "sexual difference"[all] OR "sexual
}

Figure 1: Pubmed search strategy is shown. 
were also excluded. The search also returned articles which were unrelated to the subject of interest (example: Treatment of tension pneumothorax) which were excluded as well.

Each citation was reviewed by one observer (LP) based on the inclusion and exclusion criteria, starting with title. If question of appropriateness of the study persisted, the abstract and finally the full text were reviewed prior to inclusion or exclusion of the study.

\section{Extracted imaging features}

Each eligible article was examined by one observer (LP), for imaging features that compared males and females on cross-sectional imaging, and the features were then divided into bony axes, distal femur morphology, proximal tibia morphology, patella morphology, and articular cartilage. If a study examined sex differences in multiple categories and/or subcategories, all applicable categories were assigned to that study.

\section{Analysis}

Each study was categorized as above, and anatomic features of males and females were extracted and tabulated. A descriptive analysis was performed. As the studies covered multiple categories of findings, with varied inclusion criteria, a meta-analysis could not be performed.

\section{Results}

\section{Study selection}

The literature search identified 1550 citations for consideration. A hand search yielded three additional articles satisfying inclusion criteria. Review of the article titles excluded 1104 citations, leaving 449 articles for further consideration. Finally, a full-text review excluded 365 studies, leaving 84 articles, as detailed in Figure 2. The range of sample sizes in the included investigations was as low as 17 , to as large as 1062 subjects, as shown in Table 1.

Table 1: Study characteristics and categories.

\begin{tabular}{|c|c|c|c|c|c|c|}
\hline First author & Year & Modality & Study category & Subcategory & 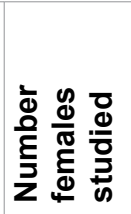 & 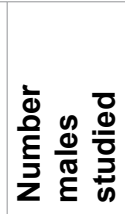 \\
\hline Ariumi [11] & 2010 & CT & Axes & Flexion, rotation & 24 & 21 \\
\hline Shabshin [9] & 2004 & MRI & Axes & Insall-salvati & 134 & 128 \\
\hline Cinotti [36] & 2013 & MRI & Axes & mPTS, IPTS & 35 & 45 \\
\hline Haddad [6] & 2012 & MRI & Axes & mPTS, IPTS & 71 & 72 \\
\hline Hudek [7] & 2011 & MRI & Axes & mPTS, IPTS & 31 & 24 \\
\hline Lustig [3] & 2013 & MRI & Axes & mPTS, IPTS & 51 & 50 \\
\hline Ristic [44] & 2014 & MRI & Axes & mPTS, IPTS & 11 & 49 \\
\hline Zhang [45] & 2014 & CT & Axes & mPTS, IPTS & 40 & 40 \\
\hline Moghtadaei [46] & 2015 & CT & Axes & Rotation & 54 & 96 \\
\hline Tao [12] & 2010 & CT & Axes & Rotation & 19 & 20 \\
\hline Raju [47] & 2015 & MRI & Axes & Rotation & 38 & 86 \\
\hline Dickschas [48] & 2016 & CT & Axes & TT-TG & 36 & 19 \\
\hline Pandit [49] & 2011 & MRI & Axes & TT-TG & 43 & 57 \\
\hline Skelley [50] & 2015 & MRI & Axes & TT-TG & 57 & 59 \\
\hline Akagi [10] & 2004 & CT & Axes & Valgus & 19 & 20 \\
\hline Han [51] & 2016 & MRI & Axes, femur & $\begin{array}{l}\text { mPTS, IPTS, AP } \\
\text { length, ML width, } \\
\text { condyle height, PO }\end{array}$ & 262 & 273 \\
\hline Van Diek [52] & 2014 & MRI & Axes, femur & $\begin{array}{l}\text { mPTS, IPTS, ML } \\
\text { width, notch }\end{array}$ & 41 & 47 \\
\hline Cinotti [21] & 2012 & MRI & Axes, femur & mPTS, IPTS, PO & 35 & 45 \\
\hline Alemparte [53] & 2007 & CT & Axes, femur & TT-TG, trochlea & 30 & 30 \\
\hline Balcarek [37] & 2010 & MRI & Axes, femur, patella & $\begin{array}{l}\text { TT-TG, trochlea, } \\
\text { patella height }\end{array}$ & 127 & 130 \\
\hline Hashemi [8] & 2008 & MRI & Axes, tibia & $\begin{array}{l}\text { mPTS, IPTS, plateau } \\
\text { depth }\end{array}$ & 33 & 22 \\
\hline
\end{tabular}




\begin{tabular}{|c|c|c|c|c|c|c|}
\hline Ding [35] & 2003 & MRI & Cartilage & G-vol & 214 & 158 \\
\hline Nishimura [54] & 2005 & MRI & Cartilage & G-vol & 31 & 37 \\
\hline Antony [55] & 2015 & MRI & Cartilage & T-vol & 155 & 173 \\
\hline Berry [56] & 2011 & MRI & Cartilage & T-vol, p-vol & 47 & 46 \\
\hline Caglar [57] & 2014 & MRI & Cartilage & $\mathrm{T} 2$ & 60 & 47 \\
\hline Joseph [58] & 2015 & MRI & Cartilage & T2 & 255 & 226 \\
\hline Mosher [59] & 2004 & MRI & Cartilage & T2 & 10 & 7 \\
\hline Eckstein [60] & 2002 & MRI & Cartilage & T2, thickness, SA & 18 & 18 \\
\hline Draper [61] & 2006 & MRI & Cartilage & Thickness & 30 & 20 \\
\hline Eckstein [62] & 2010 & MRI & Cartilage & Thickness & 597 & 465 \\
\hline Cicuttini [63] & 2002 & MRI & Cartilage & Thickness, g-vol & 96 & 70 \\
\hline Otterness [33] & 2007 & MRI & Cartilage & Thickness, SA, g-vol & 40 & 57 \\
\hline Faber [34] & 2001 & MRI & Cartilage & Thickness, SA, g-vol & 9 & 9 \\
\hline Beattie [64] & 2008 & MRI & Cartilage & Thickness, SA, t-vol & 73 & 46 \\
\hline Eckstein [65] & 2004 & MRI & Cartilage & $\begin{array}{l}\text { Thickness, SA, t-vol, } \\
\text { p-vol, f-vol }\end{array}$ & 14 & 15 \\
\hline Berry [66] & 2008 & MRI & Cartilage, patella & $\begin{array}{l}\text { P-vol, patella bone } \\
\text { volume }\end{array}$ & 186 & 111 \\
\hline Li [67] & 2012 & CT & Femur & $\begin{array}{l}\text { AP length, condyle } \\
\text { height }\end{array}$ & 39 & 51 \\
\hline $\begin{array}{l}\text { Van den Heever } \\
{[14]}\end{array}$ & 2012 & MRI & Femur & AP length, ML width & 22 & 20 \\
\hline Cavaignac [15] & 2016 & $\mathrm{CT}$ & Femur & AP length, ML width & 134 & 122 \\
\hline Cho [16] & 2015 & CT & Femur & $\begin{array}{l}\text { AP length, ML width, } \\
\text { condyle height, notch }\end{array}$ & 114 & 88 \\
\hline Pinskerova [17] & 2014 & MRI & Femur & $\begin{array}{l}\text { AP length, ML width, } \\
\text { condyle height, } \\
\text { trochlea }\end{array}$ & 100 & 100 \\
\hline Barnes [68] & 2010 & CT & Femur & $\begin{array}{l}\text { AP length, ML width, } \\
\text { condyle ratios }\end{array}$ & 39 & 27 \\
\hline Fehring [70] & 2009 & MRI & Femur & Condyle height & 100 & 112 \\
\hline Yue [71] & 2015 & CT & Femur & $\begin{array}{l}\text { Condyle height, } \\
\text { condyle ratios }\end{array}$ & 50 & 50 \\
\hline Rosenstein [72] & 2008 & MRI & Femur & Condyle ratios & 50 & 50 \\
\hline Yan [43] & 2014 & CT & Femur & $\begin{array}{l}\text { Condyle ratios, } \\
\text { trochlea }\end{array}$ & 50 & 50 \\
\hline Park [69] & 2012 & MRI & Femur & $\begin{array}{l}\text { ML width, condyle } \\
\text { ratios }\end{array}$ & 79 & 147 \\
\hline $\mathrm{Li}[73]$ & 2014 & $\begin{array}{l}\text { CT/ } \\
\text { MRI }\end{array}$ & Femur & $\begin{array}{l}\text { ML width, condyle } \\
\text { height }\end{array}$ & 65 & 96 \\
\hline Murshed [74] & 2005 & MRI & Femur & $\begin{array}{l}\text { ML width, condyle } \\
\text { height }\end{array}$ & 100 & 100 \\
\hline Vrooijink [75] & 2011 & MRI & Femur & $\begin{array}{l}\text { ML width, condyle } \\
\text { ratios, notch }\end{array}$ & 40 & 49 \\
\hline Anderson [76] & 2001 & MRI & Femur & ML width, notch & 50 & 50 \\
\hline Van Eck [77] & 2011 & MRI & Femur & Notch & 45 & 55 \\
\hline Van Eck [78] & 2010 & $\mathrm{CT}$ & Femur & Notch & 10 & 10 \\
\hline Charlton [79] & 2002 & MRI & Femur & Notch & 20 & 28 \\
\hline Dienst [80] & 2007 & MRI & Femur & Notch & 10 & 10 \\
\hline Estes [81] & 2015 & MRI & Femur & Notch & 23 & 49 \\
\hline Wang [20] & 2014 & CT & Femur & PO & 50 & 50 \\
\hline Arslan [22] & 2015 & MRI & Femur & Red marrow & 92 & 48 \\
\hline Biedert [82] & 2009 & MRI & Femur & Trochlea & 68 & 84 \\
\hline
\end{tabular}




\begin{tabular}{|c|c|c|c|c|c|c|}
\hline Hasler [83] & 2014 & MRI & Femur & Trochlea & 16 & 37 \\
\hline Kamath [84] & 2013 & MRI & Femur & Trochlea & 183 & 146 \\
\hline Wang [42] & 2012 & CT & Femur & Trochlea & 50 & 50 \\
\hline Voleti [4] & 2015 & MRI & Femur, cartilage & $\begin{array}{l}\text { Condyle height, } \\
\text { condyle ratios, PO, } \\
\text { thickness }\end{array}$ & 50 & 50 \\
\hline Lee [85] & 2015 & CT & Femur, patella & $\begin{array}{l}\text { F-SA/vol, patella } \\
\text { bone volume, patella } \\
\text { SA }\end{array}$ & 55 & 55 \\
\hline Cheng [40] & 2010 & CT & Femur, tibia & $\begin{array}{l}\text { AP length, ML width, } \\
\text { AP-t, ML-t }\end{array}$ & 78 & 94 \\
\hline Lim [18] & 2013 & MRI & Femur, tibia & $\begin{array}{l}\text { AP length, ML width, } \\
\text { AP-t, ML-t, t-ratio }\end{array}$ & 59 & 56 \\
\hline Bisson [19] & 2010 & MRI & Femur, tibia & $\begin{array}{l}\text { AP length, ML width, } \\
\text { plateau depth }\end{array}$ & 40 & 40 \\
\hline Yue [13] & 2011 & CT & Femur, tibia & $\begin{array}{l}\text { AP length, ML width, } \\
\text { trochlea, AP-t, ML-t }\end{array}$ & 20 & 20 \\
\hline Bellemans [86] & 2010 & CT & Femur, tibia & $\begin{array}{l}\text { Condyle ratio, AP-t, } \\
\text { ML-t, t-ratio }\end{array}$ & 686 & 314 \\
\hline Huang [30] & 2015 & CT & Patella & $\begin{array}{l}\text { Patella height, } \\
\text { patella width, patella } \\
\text { thickness }\end{array}$ & 60 & 60 \\
\hline Shang [41] & 2014 & CT & Patella & $\begin{array}{l}\text { Patella height, } \\
\text { patella width, patella } \\
\text { thickness }\end{array}$ & 20 & 20 \\
\hline Yoo [87] & 2007 & MRI & Patella & $\begin{array}{l}\text { Patella height, } \\
\text { patella width, patella } \\
\text { thickness }\end{array}$ & 30 & 142 \\
\hline Mahfouz [32] & 2006 & CT & Patella & Patella morphology & 95 & 133 \\
\hline Mahfouz [31] & 2007 & $\mathrm{CT}$ & Patella & Patella morphology & 95 & 133 \\
\hline Scheffel [29] & 2013 & MRI & Tibia & $A C L$ insertion site & 68 & 70 \\
\hline Kucukdurmaz [88] & 2014 & MRI & Tibia & AP-t, ML-t & 150 & 110 \\
\hline Erkocak [23] & 2016 & MRI & Tibia & AP-t, ML-t, t-ratio & 138 & 88 \\
\hline Hartel [25] & 2014 & CT & Tibia & Master shape & 38 & 79 \\
\hline Hartel [26] & 2009 & MRI & Tibia & Master shape & 110 & 127 \\
\hline Hovinga [24] & 2009 & MRI & Tibia & ML-t & 36 & 34 \\
\hline Stone [89] & 2007 & MRI & Tibia & ML-t & 38 & 63 \\
\hline Tang [27] & 2010 & MRI & Tibia & Offset & 25 & 25 \\
\hline Sun [28] & 2014 & CT & Tibia & $\begin{array}{l}\text { Posterior proximal } \\
\text { tibia }\end{array}$ & 138 & 162 \\
\hline
\end{tabular}

Legend: CT: Computed Tomography; MRI: Magnetic Resonance Imaging; TT-TG: Tibial Translation-Trochlear Groove Distance; mPTS: medial Posterior Tibial Slope; IPTS: Lateral Posterior Tibial Slope; AP: Anteroposterior; ML: Mediolateral; PO: Posterior Offset; Vol: Volume; G-vol: General volume; T-vol: Tibial volume; F-vol: Femoral volume; P-vol: Patellar volume; SA: Surface Area; F-SA: Femur Surface Area; AP-t: AP length of tibia; ML-t: ML width of tibia; T-ratio: Tibial ratio.

\section{Extracted imaging features}

The 84 included articles spanned 5 major categories shown in Table 1: Bony axes of the knee (21 papers), morphology of the distal femur (38 papers), morphology of the proximal tibia (15 papers), morphology of the patella (8 papers), and articular cartilage (16 papers). The axes category was divided into 7 subcategories (Table 2): Condylar angles, flexion, Insall-Salvati ratio (ratio of patellar length to patellar tendon length), Posterior Tibial Slope (PTS), tibial rotation, Tibial Tuberosity to Trochlear Groove (TT-TG) distance, and valgus. The distal femur category was divided into 9 subcategories
(Table 3): Anteroposterior (AP) condylar length, mediolateral (ML) condylar width, condyle height, condyle ratios, notch, posterior offset, trochlea, red marrow, and surface area/volume. The proximal tibia category was divided into 8 subcategories (Table 4): Plateau depth, AP plateau diameter, ML plateau diameter, plateau ratio, tibial offset, master shape of the proximal tibia, posterior proximal tibia, and $\mathrm{ACL}$ insertion site in the proximal tibia. The patella category was divided into 7 subcategories (Table 5): Patella height, patella width, patella thickness, patella bone volume, patella surface area, patella width to thickness ratio, patella morphology sex 


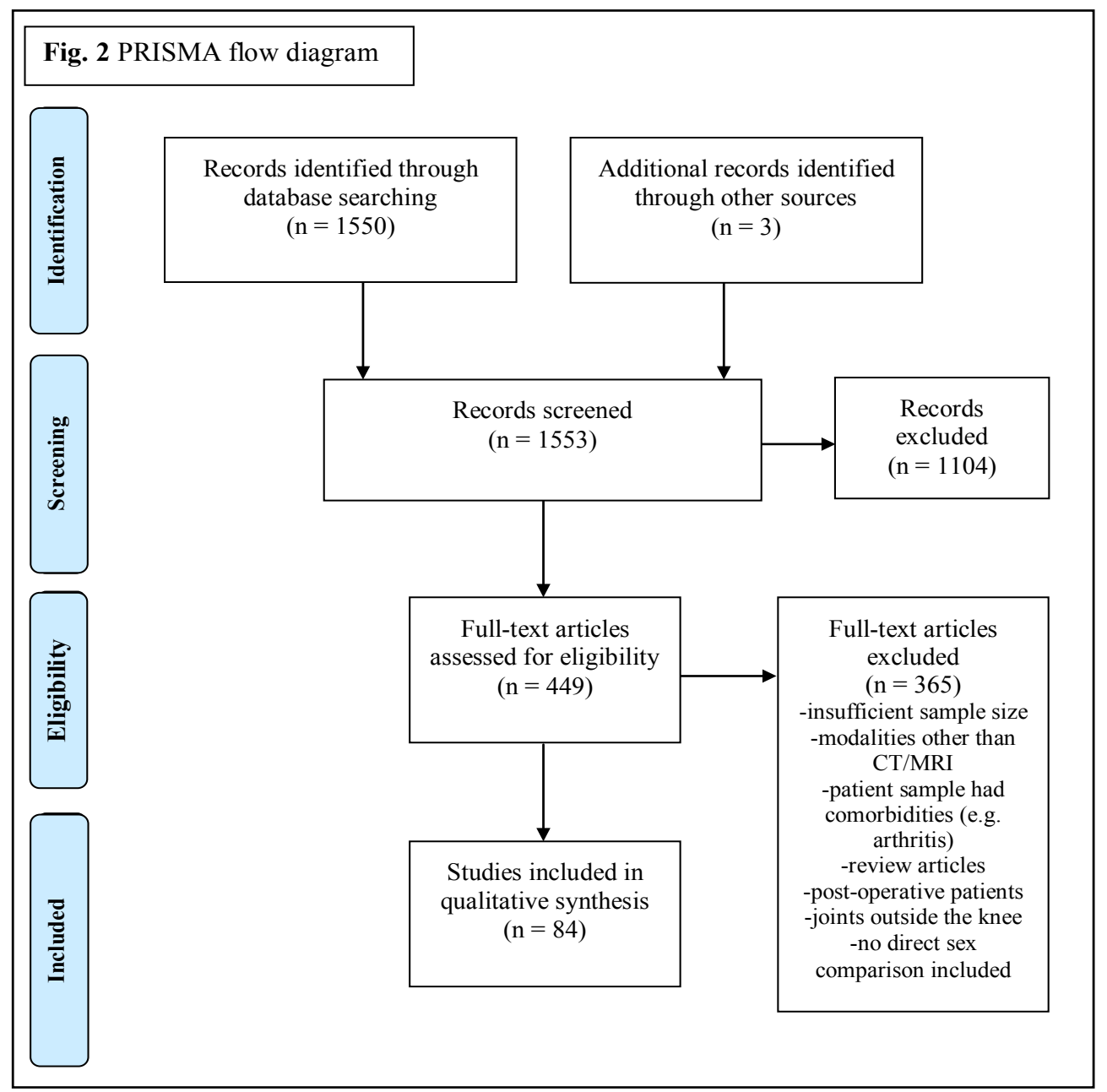

Figure 2: Diagram showing study inclusion and exclusion.

Table 2: A summary of investigations of the bony axes of the knee.

\begin{tabular}{|l|l|l|l|l|l|l|l|}
\hline First author & TT-TG & $\begin{array}{l}\text { Medial } \\
\text { PTS }\end{array}$ & Lateral PTS & Flexion & Insall-salvati & Rotation & Valgus \\
\hline Cinotti [36] & & ND & ND & & & \\
\hline Haddad [6] & & F & F & & & \\
\hline Hudek [7] & & F & ND & & & \\
\hline Lustig [3] & & ND & ND & & & \\
\hline Ristic [44] & & ND & ND & & & \\
\hline Zhang [45] & & ND & ND & & & \\
\hline Alemparte [53] & ND & & & & & \\
\hline Dickschas [48] & ND & & & & & \\
\hline Pandit [49] & ND & & & & & \\
\hline Skelley [50] & F & & & & & \\
\hline Ariumi [11] & & & & & & \\
\hline Shabshin [9] & & & & & & \\
\hline Tao [12] & & ND & ND & & & \\
\hline Akagi [10] & & ND & ND & & & \\
\hline Han [51] & & F & F & & & \\
\hline Cinotti [21] & & ND & ND & & & \\
\hline Balcarek [37] & F & & & & \\
\hline Hashemi [8] & & & & & \\
\hline Van Diek [52] & & & & & \\
\hline
\end{tabular}




\begin{tabular}{|c|c|c|c|c|c|c|c|}
\hline Moghtadaei [46] & & & & & & $\mathrm{ND}^{\mathrm{F}}$ & \\
\hline Raju [47] & & & & & & $N^{F}$ & \\
\hline SUMMARY & $\begin{array}{l}\text { Split } \\
\text { between } \\
\text { no sex } \\
\text { difference } \\
\text { and greater } \\
\text { in females }\end{array}$ & $\begin{array}{l}7 / 10 \\
\text { studies } \\
\text { say no } \\
\text { sex } \\
\text { difference }\end{array}$ & $\begin{array}{l}\text { 8/10 studies } \\
\text { say no sex } \\
\text { difference }\end{array}$ & $\begin{array}{l}\text { Males } \\
\text { exhibited } \\
\text { greater } \\
\text { degree of } \\
\text { knee flexion }\end{array}$ & $\begin{array}{l}\text { Females have } \\
\text { different normal } \\
\text { curve of Insall- } \\
\text { Salvati ratio } \\
\text { with higher } \\
\text { values }\end{array}$ & $\begin{array}{l}\text { No difference } \\
\text { in femoral } \\
\text { rotation; } \\
\text { males have } \\
\text { greater tibial } \\
\text { rotation }\end{array}$ & $\begin{array}{l}\text { Females } \\
\text { have } \\
\text { greater } \\
\text { degree } \\
\text { of knee } \\
\text { valgus }\end{array}$ \\
\hline
\end{tabular}

ND: No statistical difference; F: Greater/larger in females; M: Greater/larger in males; $\mathrm{M}^{\top}$ : Greater tibial rotation in males; NDF: No difference in femur rotation.

Table 3: A summary of investigations of the distal femur.

\begin{tabular}{|c|c|c|c|c|c|c|c|c|c|}
\hline First author & $\begin{array}{l}\text { AP con- } \\
\text { dylar } \\
\text { length }\end{array}$ & $\begin{array}{l}\text { ML width } \\
\text { (condyles, } \\
\text { EW, BW) }\end{array}$ & $\begin{array}{l}\text { Condyle } \\
\text { height }\end{array}$ & $\begin{array}{l}\text { Condyle } \\
\text { ratios }\end{array}$ & Notch & PO & $\begin{array}{l}\text { Troch- } \\
\text { lea }\end{array}$ & $\begin{array}{l}\text { Red mar- } \\
\text { row }\end{array}$ & $\begin{array}{l}\text { Femur surface } \\
\text { area/volume }\end{array}$ \\
\hline Van Diek [52] & & $M$ & & & ND & & & & \\
\hline Han [51] & $\begin{array}{l}\mathrm{M}^{\mathrm{L}} \\
\mathrm{ND}^{\mathrm{M}}\end{array}$ & $M$ & $M$ & & & $M$ & & & \\
\hline Balcarek [37] & & & & & & & $\begin{array}{l}\mathrm{F}^{1,2} \\
\mathrm{M}^{3}\end{array}$ & & \\
\hline Cinotti [21] & & & & & & $\begin{array}{l}\mathrm{F}^{\mathrm{a}, \mathrm{M}} \\
\mathrm{ND}\end{array}$ & & & \\
\hline Alemparte [53] & & & & & & & $\mathrm{F}^{1}$ & & \\
\hline Barnes [68] & $M$ & $M$ & & $\mathrm{~F}$ & & & & & \\
\hline Fehring [70] & & & $\begin{array}{l}\mathrm{M}^{\mathrm{M}} \\
\mathrm{ND}^{\mathrm{E}}\end{array}$ & & & & & & \\
\hline Li [67] & $M^{a, b}$ & & M & & & & & & \\
\hline Li [73] & & M & $M$ & & & & & & \\
\hline Rosenstein [72] & & & & $F$ & & & & & \\
\hline $\begin{array}{l}\text { Van den Heev- } \\
\text { er [14] }\end{array}$ & $M$ & M & & & & & & & \\
\hline Cho [16] & $\mathrm{M}^{\mathrm{b}}$ & $\mathrm{M}^{\mathrm{b}}$ & $\mathrm{M}^{\mathrm{b}}$ & & $\mathrm{M}^{\mathrm{b}}$ & & & & \\
\hline Vrooijink [75] & & M & & ND & ND & & & & \\
\hline Pinskerova [17] & $M$ & M & M & & & & $\begin{array}{l}N^{5} \\
M^{3,4}\end{array}$ & & \\
\hline Wang [42] & & & & $\mathrm{F}$ & & & $\mathrm{M}^{9}$ & & \\
\hline Yan [43] & & & & $M$ & & & $\mathrm{M}^{4}$ & & \\
\hline Cavaignac [15] & $M$ & $M$ & & & & & & & \\
\hline Murshed [74] & & M & M & & & & & & \\
\hline Van Eck [77] & & & & & M & & & & \\
\hline Van Eck [78] & & & & & $\begin{array}{l}\mathrm{M}^{\mathrm{NW}} \\
\mathrm{ND}^{\mathrm{NWI}}\end{array}$ & & & & \\
\hline Charlton [79] & & & & & $\begin{array}{l}M^{b} \\
N D^{a}\end{array}$ & & & & \\
\hline Park [69] & & M & $M$ & ND & M & & & & \\
\hline Yue [71] & & & M & ND & & & & & \\
\hline Dienst [80] & & & & & $M^{a}$ & & & & \\
\hline Estes [81] & & & & & $N D^{N W I}$ & & & & \\
\hline Anderson [76] & & $\begin{array}{l}\mathrm{M}^{\mathrm{b}} \\
\mathrm{ND}^{\mathrm{a}}\end{array}$ & & & $\begin{array}{l}M^{\mathrm{b}-\mathrm{NW}} \\
\mathrm{ND}^{\mathrm{a}-\mathrm{NW}} \\
\mathrm{ND}^{\mathrm{NWI}}\end{array}$ & & & & \\
\hline
\end{tabular}




\begin{tabular}{|c|c|c|c|c|c|c|c|c|c|}
\hline Wang [20] & & & & & & $\begin{array}{l}\mathrm{M}^{\mathrm{b}} \\
\mathrm{ND}^{\mathrm{a}}\end{array}$ & & & \\
\hline Arslan [22] & & & & & & & & $F$ & \\
\hline Biedert [82] & & & & & & & $M^{6}$ & & \\
\hline Hasler [83] & & & & & & & $\begin{array}{l}\mathrm{M}^{3,4} \\
\mathrm{ND}^{2}\end{array}$ & & \\
\hline Kamath [84] & & & & & & & $\mathrm{ND}^{7}$ & & \\
\hline Voleti [4] & & & $M$ & ND & & M & & & \\
\hline Lee [85] & & & & & & & & & M \\
\hline Bellemans [86] & & & & $M$ & & & & & \\
\hline Bisson [19] & $\mathrm{F}^{\mathrm{a}}$ & M & & & & & & & \\
\hline Cheng [40] & $\mathrm{M}$ & $M$ & & & & & & & \\
\hline Lim [18] & $\begin{array}{l}\mathrm{M}^{\mathrm{M}} \\
\mathrm{ND}^{\mathrm{L}}\end{array}$ & $M$ & & & & & & & \\
\hline Yue [13] & $\mathrm{M}^{\mathrm{M}, \mathrm{a}}$ & $\mathrm{M}^{\mathrm{M}, \mathrm{a}}$ & & & & & $\mathrm{ND}^{8}$ & & \\
\hline SUMMARY & $\begin{array}{l}\text { Majority } \\
\text { of studies } \\
\text { showed } \\
\text { males } \\
\text { had } \\
\text { greater } \\
\text { unadjust- } \\
\text { ed AP } \\
\text { condylar } \\
\text { dimen- } \\
\text { sions. } \\
\text { With } \\
\text { adjust- } \\
\text { ments, } 2 \\
\text { studies } \\
\text { demon- } \\
\text { strate } \\
\text { contro- } \\
\text { versy. }\end{array}$ & $\begin{array}{l}\text { Greater } \\
\text { ML condy- } \\
\text { lar width } \\
\text { in males, } \\
\text { although } \\
\text { one paper } \\
\text { suggested } \\
\text { difference } \\
\text { does not } \\
\text { persist after } \\
\text { adjustment }\end{array}$ & $\begin{array}{l}\text { Majority } \\
\text { show } \\
\text { that } \\
\text { males } \\
\text { have } \\
\text { greater } \\
\text { condylar } \\
\text { height }\end{array}$ & $\begin{array}{l}\text { No con- } \\
\text { sensus } \\
\text { about } \\
\text { condylar } \\
\text { size/ } \\
\text { aspect } \\
\text { ratios, } \\
\text { although } \\
\text { some } \\
\text { papers } \\
\text { focused } \\
\text { on unique } \\
\text { ratios } \\
\text { or mea- } \\
\text { sured } \\
\text { metrics } \\
\text { differently } \\
\text { than the } \\
\text { others }\end{array}$ & $\begin{array}{l}\text { Males } \\
\text { had } \\
\text { greater } \\
\text { notch } \\
\text { widths, } \\
\text { although } \\
\text { major- } \\
\text { ity of } \\
\text { studies } \\
\text { showed } \\
\text { this dif- } \\
\text { ference } \\
\text { did not } \\
\text { persist } \\
\text { after } \\
\text { adjust- } \\
\text { ment/ } \\
\text { when } \\
\text { evaluat- } \\
\text { ing NWI } \\
\text { rather } \\
\text { than } \\
\text { simple } \\
\text { NW }\end{array}$ & $\begin{array}{l}\text { Males } \\
\text { had } \\
\text { greater } \\
\text { offset } \\
\text { with no } \\
\text { adjust- } \\
\text { ment; } \\
\text { with } \\
\text { adjust- } \\
\text { ment, no } \\
\text { differ- } \\
\text { ence or } \\
\text { maybe } \\
\text { slightly } \\
\text { greater } \\
\text { in fe- } \\
\text { males }\end{array}$ & $\begin{array}{l}\text { Overall, } \\
\text { findings } \\
\text { suggest } \\
\text { greater } \\
\text { tendency } \\
\text { towards } \\
\text { trochlear } \\
\text { dyspla- } \\
\text { sia (met- } \\
\text { rics re- } \\
\text { lating to } \\
\text { shallow } \\
\text { sulcus } \\
\text { depth/ } \\
\text { asym- } \\
\text { metry), } \\
\text { although } \\
\text { some } \\
\text { charac- } \\
\text { teristics } \\
\text { showed } \\
\text { no sex } \\
\text { differ- } \\
\text { ence }\end{array}$ & & \\
\hline
\end{tabular}

ND: No statistical difference; F: Greater/larger in females; M: Greater/larger in males; EW/BW: Epicondylar width/bicondylar width; $X^{\mathrm{a}}$ : With adjustments (such as weight, height, BMI, bone length); $\mathrm{X}^{\mathrm{b}}$ : Without adjustments (such as weight, height, BMI, bone length); $X^{\mathrm{M}}$ : Medial only; $\mathrm{X}^{\mathrm{P}}$ : Patella only; $\mathrm{X}^{\mathrm{L}}$ : Lateral only; $\mathrm{X}^{\mathrm{E}}$ : Elsewhere; $\mathrm{X}^{1}$ : Sulcus angle; $\mathrm{X}^{2}$ : Trochlear asymmetry; $\mathrm{X}^{3}$ : Trochlear depth; $X^{4}$ : Trochlear and/or trochlear facet width; $X^{5}$ : Trochlear shape ratios (trochlear depth relative to trochlear width); $X^{6}$ : Trochlear height ratios (AP length of medial condyle relative to bicondylar width); $X^{7}$ : Trochlear inclination; $X^{8}$ : Trochlear groove orientation/location; $X^{9}$ : Trochlear groove 3D modeling with radius and arc length.

Table 4: A summary of investigations of the proximal tibia.

\begin{tabular}{|c|c|c|c|c|c|c|c|c|}
\hline First author & $\begin{array}{l}\text { Plateau } \\
\text { depth }\end{array}$ & $\begin{array}{l}\text { AP } \\
\text { plateau }\end{array}$ & $\begin{array}{l}\text { ML } \\
\text { plateau }\end{array}$ & Plateau ratio & Offset & $\begin{array}{l}\text { Master } \\
\text { shape }\end{array}$ & $\begin{array}{l}\text { Posterior } \\
\text { prox tibia }\end{array}$ & $\begin{array}{l}\text { ACL insertion } \\
\text { site }\end{array}$ \\
\hline Hashemi [8] & $\mathrm{ND}^{\mathrm{M}}$ & & & & & & & \\
\hline Bellemans [86] & & M & M & $\begin{array}{l}\mathrm{F} \\
\mathrm{ND}\end{array}$ & & & & \\
\hline Bisson [19] & $F$ & & & & & & & \\
\hline Cheng [40] & & $\mathrm{M}^{\mathrm{M}}$ & $\mathrm{M}^{\mathrm{M}}$ & & & & & \\
\hline Lim [18] & & $M$ & $M$ & $M$ & & & & \\
\hline Yue [13] & & $\begin{array}{l}M^{a} \\
N^{b}\end{array}$ & $\begin{array}{l}\mathrm{M}^{\mathrm{a}} \\
\mathrm{ND}^{\mathrm{b}}\end{array}$ & & & & & \\
\hline Tang [27] & & & & & $\mathrm{M}^{1}$ & & & \\
\hline
\end{tabular}




\begin{tabular}{|c|c|c|c|c|c|c|c|c|}
\hline Erkocak [23] & & M & M & M & & & & \\
\hline Hartel [25] & & & & & & $\mathrm{ND}^{2}$ & & \\
\hline Hartel [26] & & & & & & $\mathrm{ND}^{2}$ & & \\
\hline Hovinga [24] & & & M & & & & & \\
\hline Kucukdurmaz [88] & & ND & & & & & & \\
\hline Stone [89] & & & $M^{a, b}$ & & & & & \\
\hline Sun [28] & & & & & & & $\mathrm{ND}^{3}$ & \\
\hline Scheffel [29] & & & & & & & & $\mathrm{ND}^{4}$ \\
\hline SUMMARY & $\begin{array}{l}1 / 2 \text { studies } \\
\text { say no } \\
\text { difference, } \\
1 / 2 \text { showed } \\
\text { greater } \\
\text { depth in } \\
\text { females }\end{array}$ & $\begin{array}{l}\text { Majority } \\
\text { show } \\
\text { greater } \\
\text { AP } \\
\text { plateau } \\
\text { depth in } \\
\text { males }\end{array}$ & $\begin{array}{l}\text { Majority } \\
\text { show } \\
\text { greater } \\
\text { ML } \\
\text { plateau } \\
\text { width in } \\
\text { males }\end{array}$ & $\begin{array}{l}2 / 3 \text { studies } \\
\text { showed greater } \\
\text { aspect ratios } \\
\text { in males; } 1 \\
\text { showed greater } \\
\text { or no difference } \\
\text { in females }\end{array}$ & $\begin{array}{l}\text { Greater } \\
\text { offset } \\
\text { in } \\
\text { males }\end{array}$ & $\begin{array}{l}\text { No sex } \\
\text { difference } \\
\text { in "master } \\
\text { shape" of } \\
\text { proximal } \\
\text { tibia }\end{array}$ & $\begin{array}{l}\text { No sex } \\
\text { difference in } \\
\text { posterior arc/ } \\
\text { radius }\end{array}$ & $\begin{array}{l}\text { No sex } \\
\text { difference in } \\
\text { tibial insertion } \\
\text { site of } A C L\end{array}$ \\
\hline
\end{tabular}

ND: No statistical difference; F: Greater/larger in females; M: Greater/larger in males; $X^{a}$ : With adjustments (such as weight, height, BMI, bone length); $\mathrm{X}^{\mathrm{b}}$ : Without adjustments (such as weight, height, BMI, bone length); $\mathrm{X}^{\mathrm{M}}$ : Medial only; $\mathrm{X}^{1}$ : Anterolateral tibial shaft offset relative to tibial plateau; $X^{2}$ : Master shape of tibia plateau (model); $X^{3}$ : Posterior proximal tibia arc angle/radius; $\mathrm{X}^{4}$ : Tibial ACL insertion site as $\%$ of total AP tibial plateau dimension.

Table 5: A summary of investigations of the patella.

\begin{tabular}{|c|c|c|c|c|c|c|c|}
\hline First author & $\begin{array}{l}\text { Patella } \\
\text { height }\end{array}$ & $\begin{array}{l}\text { Patella } \\
\text { width }\end{array}$ & $\begin{array}{l}\text { Patella } \\
\text { thickness }\end{array}$ & $\begin{array}{l}\text { Patella } \\
\text { bone } \\
\text { volume }\end{array}$ & Patella SA & $\begin{array}{l}\text { Patella width } \\
\text { to thickness } \\
\text { ratio }\end{array}$ & $\begin{array}{l}\text { Patella } \\
\text { morphology sex } \\
\text { model }\end{array}$ \\
\hline Balcarek [37] & ND & & & & & & \\
\hline Berry [66] & & & & M & & & \\
\hline Lee [85] & & & & M & $M$ & & \\
\hline Huang [30] & $M^{a}$ & $M^{a}$ & $M^{a}$ & & & ND & \\
\hline Mahfouz [32] & & & & & & & $93.51 \%$ accurate \\
\hline Mahfouz [31] & & & & & & & $96 \%$ accurate \\
\hline Shang [41] & $M$ & M & $M$ & & & & \\
\hline Yoo [87] & $M$ & $M$ & $M$ & & & & \\
\hline SUMMARY & $\begin{array}{l}\text { Majority } \\
\text { show } \\
\text { greater } \\
\text { height in } \\
\text { males }\end{array}$ & $\begin{array}{l}\text { All show } \\
\text { greater width } \\
\text { in males, } \\
\text { even after } \\
\text { adjustment }\end{array}$ & $\begin{array}{l}\text { All show } \\
\text { greater } \\
\text { thickness } \\
\text { in males, } \\
\text { even after } \\
\text { adjustment }\end{array}$ & $\begin{array}{l}\text { Greater in } \\
\text { males }\end{array}$ & $\begin{array}{l}\text { Greater in } \\
\text { males }\end{array}$ & $\begin{array}{l}\text { No sex } \\
\text { difference }\end{array}$ & $\begin{array}{l}\text { 3d modeling } \\
\text { has high sex } \\
\text { identification } \\
\text { accuracy }\end{array}$ \\
\hline
\end{tabular}

ND: No statistical difference; F: Greater/larger in females; M: Greater/larger in males; $\mathrm{X}^{\mathrm{a}}$ : With adjustments (such as weight, height, $\mathrm{BMI}$, bone length); $\mathrm{X}^{\mathrm{b}}$ : Without adjustments (such as weight, height, BMI, bone length).

Table 6: A summary of investigations of the articular cartilage.

\begin{tabular}{|c|c|c|c|c|c|c|c|}
\hline First author & T2 value & Thickness & SA & $\begin{array}{l}\text { General } \\
\text { volume }\end{array}$ & $\begin{array}{l}\text { Tibial } \\
\text { volume }\end{array}$ & $\begin{array}{l}\text { Patellar } \\
\text { volume }\end{array}$ & $\begin{array}{l}\text { Femur } \\
\text { volume }\end{array}$ \\
\hline Caglar [57] & ND & & & & & & \\
\hline Joseph [58] & $\begin{array}{l}\text { FMF } \\
N^{E}\end{array}$ & & & & & & \\
\hline Mosher [59] & ND & & & & & & \\
\hline Draper [61] & & M & & & & & \\
\hline Eckstein [62] & & M & & & & & \\
\hline Eckstein [60] & ND & ND & M & & & & \\
\hline Antony [55] & & & & & M & & \\
\hline Berry [56] & & & & & M & M & \\
\hline Ding [35] & & & & $M$ & & & \\
\hline
\end{tabular}




\begin{tabular}{|c|c|c|c|c|c|c|c|}
\hline Nishimura [54] & & & & $\begin{array}{l}\mathrm{M}^{\mathrm{b}} \\
\mathrm{ND}^{\mathrm{a}}\end{array}$ & & & \\
\hline Cicuttini [63] & & $\mathrm{M}^{\mathrm{a}}$ & & $M^{a}$ & & & \\
\hline Otterness [33] & & $M^{a}$ & $M^{a}$ & $\mathrm{M}^{\mathrm{a}}$ & & & \\
\hline Beattie [64] & & M & M & & M & & \\
\hline Eckstein [65] & & $\mathrm{ND}^{\mathrm{P}, \mathrm{LT}}$ & M & & M & M & M \\
\hline Faber [34] & & $\begin{array}{l}\mathrm{M}^{\mathrm{b}} \\
\mathrm{ND}^{\mathrm{a}}\end{array}$ & $\begin{array}{l}M^{b} \\
N D^{a}\end{array}$ & $\begin{array}{l}\mathrm{M}^{\mathrm{b}} \\
\mathrm{ND}^{\mathrm{a}}\end{array}$ & & & \\
\hline Berry [66] & & & & & & M & \\
\hline SUMMARY & $\begin{array}{l}\text { Majority show no sex } \\
\text { difference }\end{array}$ & $\begin{array}{l}\text { Most show } \\
\text { greater } \\
\text { thickness } \\
\text { in males, } \\
\text { although } \\
\text { 3/8 studies } \\
\text { suggest no } \\
\text { difference }\end{array}$ & $\begin{array}{l}\text { Mostly show } \\
\text { greater } \\
\text { surface area } \\
\text { in males, } \\
\text { although } \\
\text { controversial } \\
\text { after } \\
\text { adjustment }\end{array}$ & $\begin{array}{l}\text { Greater in } \\
\text { males without } \\
\text { adjustment; } \\
50 \% \text { say } \\
\text { greater in } \\
\text { males with } \\
\text { adjustment vs. } \\
\text { no difference }\end{array}$ & $\begin{array}{l}\text { Greater in } \\
\text { males }\end{array}$ & $\begin{array}{l}\text { Greater in } \\
\text { males }\end{array}$ & $\begin{array}{l}\text { Greater in } \\
\text { males }\end{array}$ \\
\hline
\end{tabular}

ND: No statistical difference; F: Greater/larger in females; M: Greater/larger in males; $X^{a}$ : With adjustments (such as weight, height, BMI, bone length); $X^{b}$ : Without adjustments (such as weight, height, BMI, bone length); $X^{M F}$ : Medial femur only; $X^{P}$ : Patella only; $\mathrm{X}^{\mathrm{LT}}$ : Lateral tibia only; $\mathrm{X}^{\mathrm{E}}$ : Elsewhere.

model (3D-CT modeling for forensic sex analysis with a single patella). The articular cartilage category was divided into 7 subcategories (Table 6): T2 cartilage values, thickness, surface area, general volume, tibial volume, patellar volume, and femur volume.

\section{Analysis}

Bony axes of the knee: Although there were some common results, most of the metrics studied in articles investigating bony axes of the knee showed controversy with regards to sex differences, and some articles investigated unique metrics not evaluated in other articles. Studies evaluating the TT-TG showed either no sex difference ( $3 / 5$ studies), or greater values in females, particularly with a history of patellar instability (2/5 studies showing $14.1-19.5 \mathrm{~mm}$ in females vs. $12.6-17.0 \mathrm{~mm}$ in males). The majority of studies examining PTS showed no sex difference in either the medial (7/10 studies) or lateral tibial plateau (8/10 studies), although the remaining studies showed a greater slope in females $(2 / 10$ studies for lateral tibial plateau; $3 / 10$ studies for medial tibial plateau), with females having a mean medial PTS ranging from 4.9 - 6.3 degrees compared to $3.0-5.1$ degrees in males [6-8], and a mean lateral PTS ranging from $6.3-7.0$ degrees in females compared with $4.8-5.4$ degrees in males $[6,8]$. Females had greater average Insall-Salvati ratios (1.0878 in females vs. 1.0032 in males) and valgus angles at the knee (mean of 5.3 degrees in females vs. 3.1 degrees in males), although these metrics were only described in one study each $[9,10]$. Males exhibited a greater degree of flexion at the knee, also only described in one study [11]. Femoral rotation was described in three studies, which showed no sex difference; however, one study showed greater tibial rotation in males [12].
Morphology of the distal femur: This group of studies had the largest volume of articles and metrics investigated. Examples of distal femur metrics studied are seen in Figure 3. In nearly all femoral size metrics (including metrics such as epicondylar width, AP diameter of each condyle, height of the condyles, surface area of the distal femur etc.), males had greater unadjusted values. For example, unadjusted measurements of the AP dimensions of the medial condyle ranged from $55-64 \mathrm{~mm}$ in females with significantly greater dimensions in males in most papers, ranging from $61-70 \mathrm{~mm}$ [13-18]. Unadjusted AP measurements of the lateral condyle were also greater in males, ranging from $58-66 \mathrm{~mm}$ in females compared with 65-72 $\mathrm{mm}$ in males [13-17]. However, when adjustments for height, weight, femoral length, leg length, etc., were performed, many of these sex differences decreased or disappeared, or disagreement existed within the investigations. For example, one study showed statistically greater mean AP medial femoral condyle measurements in females ( $77 \mathrm{~mm}$ compared to $74 \mathrm{~mm}$ ) and greater mean AP lateral femoral condyle measurements in males (79 $\mathrm{mm}$ compared to $74 \mathrm{~mm}$ ) when normalized to the transepicondylar width of the femur [19]. Condylar aspect ratios were also controversial, with some studies showing no sex difference, some showing greater aspect ratios in males, and some showing greater aspect ratios in females. Studies also looked specifically at the intercondylar notch and found that while males have greater unadjusted notch widths, when using the notch width index or adjusting for other factors, the sex difference often decreased or disappeared.

Three studies looked at posterior femoral offset and two thirds of the studies found greater femoral offset in males. Two of the studies looked at posterior 

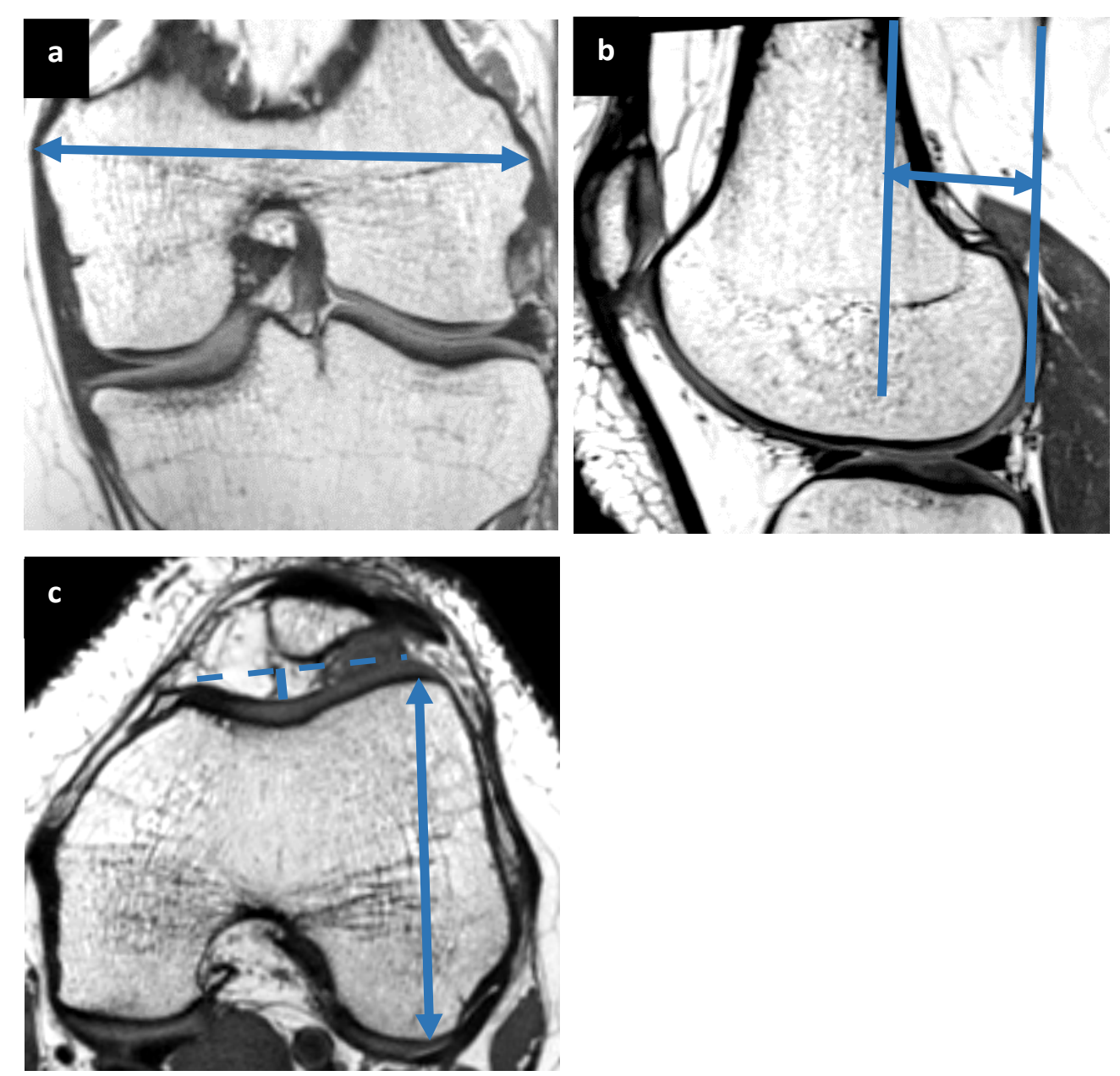

Figure 3: (a) Example figures showing distal femur metrics. Transepicondylar width is an example of a mediolateral (ML) metric; (b) Posterior offset of the femur; (c) Trochlear depth (solid line) and lateral condyle height (solid arrow).

offset as a ratio to femoral size; however, one study calculated the ratio based on AP diameter of the distal femoral condyles, finding no sex difference, while the other study used the diameter of the femoral diaphysis, finding the medial condyle ratio to be greater in females, but not different in the lateral compartment $[20,21]$.

The trochlea was evaluated in 10 different studies, which showed a general trend (with some exceptions) towards more metrics suggesting trochlear dysplasia in females (such as shallower sulcus/greater sulcus angle, or greater asymmetry). One final unique study evaluated anatomical sex differences in the femur, finding greater residual red marrow in females than males [22].

Morphology of the proximal tibia: The majority of studies showed greater proximal tibial metrics in males (such as plateau depth, and AP/ML diameter of the tibia), often persisting with adjustment for body height and weight. For example, unadjusted mediolateral tibial plateau widths were larger in males, measuring 77-81 $\mathrm{mm}$ in males compared to $69-70 \mathrm{~mm}$ in females $[23,24]$. Tibial plateau ratios were less conclusive, with $2 / 3$ studies showing greater or no difference between sexes.

There were 5 studies evaluating unique sex dif- ferences of the proximal tibia: Two studies were performed by the same group at the same institution which evaluated a master shape of the proximal tibia produced through a contour detection algorithm, finding no sex differences $[25,26]$. One study looked at the anterolateral offset of the tibial shaft relative to the tibial plateau, which was greater in males [27]. Another study evaluated the morphology of the posterior aspect of the proximal tibia, finding no sex difference in either the arc angle or radius of the arc forming the posterior contour [28]. Finally, another study calculated the tibial insertion site of the ACL as a percentage of the AP tibial plateau dimension and found that there were no significant sex differences in this percentage [29].

Morphology of the patella: The majority of studies evaluating the patella showed greater metrics in males, which usually persisted after adjustment for height and weight. However, one paper evaluated the patella width to thickness ratio and found no sex difference [30]. Interestingly, two studies from the same institution looked at 3D forensic modeling using CT and found that the patellar morphology was able to be modeled with at least $93 \%$ accuracy when predicting sex of the donor [31,32]. 
Articular cartilage: We found articles that discussed T2 cartilage metrics (thickness, area, and volumes) as well as T2 signal characteristics. Not all studies adjusted findings for patient height and weight. Males had greater cartilage size metrics when no adjustments were made. When adjusting for height and weight, two studies found that females had less cartilage volume, thickness, and joint surface area, while two other studies found no sex discrepancy in cartilage metrics. For example, in two studies that showed statistically greater total knee cartilage volume in males (mean ranging from $23-27 \mathrm{cc}$ ) compared to females (mean $18 \mathrm{cc}$ ) [33,34], statistical significance was lost after adjusting for patient height/ weight [34]. The other study showed greater cartilage volume in males, to a lesser degree after normalizing to weight (male to female volume difference decreasing from $9 \mathrm{cc}$ to $5 \mathrm{cc}$ ) or height (difference decreasing from $9 \mathrm{cc}$ to $7 \mathrm{cc}$ ) [33]. Of potential importance, one of the two studies which showed the sex difference also described a faster cartilage loss in females with aging relative to males, and this was not evaluated in other studies in our sample [35]. Finally, four studies looked at sex differences in T2 signal characteristics, and only one found statistically significant higher T2 values in females, only in the medial femur cartilage.

\section{Discussion}

This was the first report comprehensively assessing the existing literature to identify differences in the osseous and cartilage structures of the knee between the sexes. While sex differences in the knee had been previously documented, they had largely focused on the $A C L$ anatomy, injury rates and outcomes. The sex differences of bone and cartilage were less well understood, and this comprehensive review demonstrated that there were some well-established sex differences, some trends toward differences in anatomy, and that other sex differences still require further investigation.

As women's sports medicine is still an emerging field, we uncovered investigations of anatomic sex differences that utilized varied and non-uniform anatomic metrics and study methods. In particular, many of the studies did not normalize the metrics to patient size (such as patient height, weight, femur length etc), or did not normalize the metrics to patient size in a standard way (such as to a particular bone diameter). In addition, in some studies, sex comparisons were secondary objectives rather than the primary endpoint of the investigation, perhaps resulting in a study design that less optimally assessed sex differences. Another factor that might explain some of the varied results observed was the potential for measurement error in many of the metrics utilized; whether measurements were made by hand or with an automated tool, inter-observer reliability statistics were not available in many investigations. Hence, this comprehensive review yielded a hetero- geneous group of studies, upon which a meta-analysis could not be performed; rather, we aimed to accurately summarize current knowledge and gaps in information.

Overall, the literature indicated that unadjusted size metrics in the knee, including various femoral, tibial, patellar, and cartilage metrics, were larger in males than females. However, once adjustments were made for factors such as subject height, weight, and total femur length, some of the sex differences in anatomic metrics did not persist. This phenomenon was particularly true in many distal femur, proximal tibia, and articular cartilage metrics. Thus, adjusting for body size factors was critical to determining whether observed sex differences were innate, or whether they were due solely to the patient size.

The majority of studies showed no sex differences in measurements of the bony axes of the knee, although in studies where a difference was observed, females were shown to have larger metrics (including in the TT-TG, the Insall-Salvati, the PTS PTS, and the valgus angulation). Alterations in such metrics had been posed as potential risk factors for sports injuries in females. For example, sex differences in the PTS were thought to be a risk factor in developing $A C L$ and meniscus tears, due to accompany altered biomechanics [36]. Given the known female predilection for ACL tears, such investigations of anatomic differences in the PTS may provide important insight into why this female predilection existed, and when designing a treatment plan, anatomic differences might play an important role in therapy and future prevention.

Another area of interest that was explored in this study was the trochlear morphology. Unfortunately, the studies found in our search were fewer in number and heterogeneous, resulting in limited conclusive power. Included studies that analyzed trochlear morphology suggested a trend towards a more shallow trochlea in females with lesser trochlear depth, greater sulcus angle, and greater asymmetry in the trochlea, all important anatomic features to further exploring the diagnosis of trochlear dysplasia and its predisposition to patellar maltracking. Our findings regarding the trochlea were therefore consistent with observations in sports medicine, that females had higher rates of patellar instability (including patellofemoral pain and sensation of patellar subluxation), higher rates of primary dislocation during the high-risk adolescent period (age 10-17), and higher rates of recurrent dislocations in patients with instability symptoms $[37,38]$.

Other than the trochlea, most measures of the distal femur, when adjusting for height, weight and other confounding factors, were not significantly different between males and females (including aspect ratios and notch width). However, one measure that reported important in the assessment of knee arthroplasty functionality was the posterior femoral offset. This measure- 
ment was found to be greater in males than females in two of three anatomic studies. Hence, customized arthroplasties designed with attention to the offset were probably important to subsequent restoration of function in patients requiring surgery, and care was often taken to consider this factor in treatment planning [39].

In the patella, it appeared that sex differences likely persisted after adjusting for confounding factors, with males had generally larger and exaggerated morphology compared to females. Additionally, it was very interesting that the sex differences of the patella were enough that a trained neural network in forensic CT modeling can identify patient sex with a high degree of accuracy $[32,32]$. These findings might have clinical implications to both developing injury (also potentially contributing to patellar instability/dislocation in addition to trochlear morphology), and to arthroplasty design and surgical treatment. Similarly, in the tibia, males tended to have larger tibias with more pronounced features (such as greater depth or greater anteroposterior to lateral diameter). These proximal tibial differences likely had clinical implications, with resulting alterations to the biomechanics and stability of the knee joint, shear forces (and therefore injury risk) on the intra-articular soft tissues such as cruciate ligaments, articular cartilage and menisci might be different between the sexes as a consequence.

Our comprehensive review had limitations. We excluded studies that were not written in the English language, which limited the scope of our search. We included studies focusing on certain ethnic/racial populations, such as studies looking specifically at Chinese subjects only $[12,20,27,28,40-44]$. Such a specific patient population focus might limit the generalizability of the findings; we did not adjust for ethnicity, race or age, as not enough data was available to do so. Also, as already mentioned, the study populations, research methodology and outcome measures were highly variable between investigations; due to the heterogeneity of studies, a quality assessment tool could not be utilized to capture quality differences and a meta-analysis could not be performed. In addition, measurement from different studies made by different modalities could influence the results, but there was no inter-modality comparison available to determine the accuracy and interplay between modalities.

In conclusion, while a robust understanding of the bony and cartilaginous sex differences in the knee were still emerging, we had provided a compendium of current knowledge in this important field. Such information was valuable to potentially providing a customized radiology interpretation for individual patients of different sexes and identifying patients at risk of injury. In this way, imaging metrics of sex differences might help promote better surgical outcomes and post-operative care, as an understand- ing of the sex differences continues to be elucidated. Finding metrics that had no sex difference would also be beneficial in directing resources away from sex-specific approaches in sports medicine, to those that were influenced by patient sex. Radiology was uniquely positioned to study these anatomic differences in a noninvasive manner with continually improving imaging techniques, including high-resolution and increasingly fast acquisitions, which can improve the standardization of measurements and findings. Moving forward, investigations looking at sex differences should conduct rigorous, reproducible measurements that were normalized to patient height, weight, and femur length at a minimum, so that any sex differences discovered can be attributable to sex rather than patient size alone.

\section{Acknowledgments}

Individuals who are not authors: We thank Katie Lobner, clinical informationist at the Johns Hopkins Welch Medical library, for her assistance in developing/refining our search strategy and assistance in collecting the articles reviewed for this project.

\section{Grants/Funding}

None.

\section{References}

1. Arendt E, Dick R (1995) Knee injury patterns among men and women in collegiate basketball and soccer. NCAA data and review of literature. Am J Sports Med 23: 694-701.

2. Terauchi M, Hatayama K, Yanagisawa S, Saito K, Takagishi K (2011) Sagittal alignment of the knee and its relationship to noncontact anterior cruciate ligament injuries. Am J Sports Med 39: 1090-1094.

3. Lustig S, Scholes CJ, Leo SP, Coolican M, Parker DA (2013) Influence of soft tissues on the proximal bony tibial slope measured with two-dimensional MRI. Knee Surg Sports Traumatol Arthrosc 21: 372-379.

4. Voleti PB, Stephenson JW, Lotke PA, Lee GC (2015) No sex differences exist in posterior condylar offsets of the knee. Clin Orthop Relat Res 473: 1425-1431.

5. Merchant AC, Arendt EA, Dye SF, Fredericson M, Grelsamer RP, et al. (2008) The female knee: anatomic variations and the female-specific total knee design. Clin Orthop Relat Res 12: 3059-3065.

6. Haddad B, Konan S, Mannan K, Scott G (2012) Evaluation of the posterior tibial slope on MR images in different population groups using the tibial proximal anatomical axis. Acta Orthop Belg 78: 757-763.

7. Hudek R, Fuchs B, Regenfelder F, Koch PP (2011) Is noncontact ACL injury associated with the posterior tibial and meniscal slope? Clin Orthop Relat Res 469: 2377-2384.

8. Hashemi J, Chandrashekar N, Gill B, Beynnon BD, Slauterbeck JR, et al. (2008) The geometry of the tibial plateau and its influence on the biomechanics of the tibiofemoral joint. $J$ Bone Joint Surg Am 90: 2724-2734.

9. Shabshin N, Schweitzer ME, Morrison WB, Parker L (2004) MRI criteria for patella alta and baja. Skeletal Radiol 33: 445-450. 
10. Akagi M, Oh M, Nonaka T, Tsujimoto $H$, Asano $T$, et al. (2004) An anteroposterior axis of the tibia for total knee arthroplasty. Clin Orthop Relat Res 213-219.

11. Ariumi A, Sato T, Kobayashi K, Koga Y, Omori G, et al. (2010) Three-dimensional lower extremity alignment in the weight-bearing standing position in healthy elderly subjects. J Orthop Sci 15: 64-70.

12. Tao K, Cai M, Li SH (2010) The anteroposterior axis of the tibia in total knee arthroplasty for chinese knees. Orthopedics 33: 799.

13. Yue B, Varadarajan KM, Ai S, Tang T, Rubash HE, et al. (2011) Gender differences in the knees of Chinese population. Knee Surg Sports Traumatol Arthrosc 19: 80-88.

14. van den Heever DJ, Scheffer C, Erasmus P, Dillon E (2012) Classification of gender and race in the distal femur using self organising maps. Knee 19: 488-492.

15. Cavaignac E, Savall F, Faruch M, Reina N, Chiron $P$, et al. (2016) Geometric morphometric analysis reveals sexual dimorphism in the distal femur. Forensic Sci Int 259: 246.

16. Cho HJ, Kwak DS, Kim IB (2015) Morphometric Evaluation of Korean Femurs by Geometric Computation: Comparisons of the Sex and the Population. Biomed Res Int.

17. Pinskerova V, Nemec K, Landor I (2014) Gender differences in the morphology of the trochlea and the distal femur. Knee Surg Sports Traumatol Arthrosc 22: 2342-2349.

18. Lim HC, Bae JH, Yoon JY, Kim SJ, Kim JG, et al. (2013) Gender differences of the morphology of the distal femur and proximal tibia in a Korean population. Knee 20: 26-30.

19. Bisson LJ, Gurske-DePerio J (2010) Axial and sagittal knee geometry as a risk factor for noncontact anterior cruciate ligament tear: a case-control study. Arthroscopy 26: 901906.

20. Wang W, Tsai TY, Yue B, Kwon YM, Li G (2014) Posterior femoral condylar offsets of a Chinese population. Knee 21: 553-556.

21. Cinotti G, Sessa P, Ripani FR, Postacchini R, Masciangelo $\mathrm{R}$, et al. (2012) Correlation between posterior offset of femoral condyles and sagittal slope of the tibial plateau. J Anat 221: $452-458$

22. Arslan G, Ozmen E, Soyturk M (2015) MRI of Residual Red Bone Marrow in the Distal Femur of Healthy Subjects. Pol J Radiol 80: 300-304.

23. Erkocak OF, Kucukdurmaz F, Sayar S, Erdil ME, Ceylan $\mathrm{HH}$, et al. (2016) Anthropometric measurements of tibial plateau and correlation with the current tibial implants. Knee Surg Sports Traumatol Arthrosc 24: 2990-2997.

24. Hovinga KR, Lerner AL (2009) Anatomic variations between Japanese and Caucasian populations in the healthy young adult knee joint. J Orthop Res 27: 1191-1196.

25. Hartel MJ, Loosli Y, Delfosse D, Diel P, Thali M, et al. (2014) The influence of tibial morphology on the design of an anatomical tibial baseplate for TKA. Knee 21: 415-419.

26. Hartel MJ, Loosli Y, Gralla J, Kohl S, Hoppe S, et al. (2009) The mean anatomical shape of the tibial plateau at the knee arthroplasty resection level: an investigation using MRI. Knee 16: 452-457.

27. Tang Q, Zhou Y, Yang D, Xu H, Liu Q (2010) The offset of the tibial shaft from the tibial plateau in Chinese people. $\mathrm{J}$ Bone Joint Surg Am 92: 1981-1987.

28. Sun H, Luo CF, Shi HP, Yang G, Zhong B, et al. (2014) Morphological measurements of the posterior surface of the normal proximal tibia in a healthy Chinese population. Knee 21: $567-572$

29. Scheffel PT, Henninger HB, Burks RT (2013) Relationship of the intercondylar roof and the tibial footprint of the $A C L$ : implications for ACL reconstruction. Am J Sports Med 41: 396-401.

30. Huang $A B$, Luo $X$, Song $C H$, Zhang JY, Yang $Y Q$, et al. (2015) Comprehensive assessment of patellar morphology using computed tomography-based three-dimensional computer models. Knee 22: 475-480.

31. Mahfouz M, Badawi A, Merkl B, Fatah EE, Pritchard E, et al. (2007) Patella sex determination by 3D statistical shape models and nonlinear classifiers. Forensic Sci Int 173: 161170.

32. Mahfouz M, Badawi A, Merkl B, Fatah EE, Pritchard E, et al. (2006) 3D statistical shape models of patella for sex classification. Conf Proc IEEE Eng Med Biol Soc 1: 3439-3445.

33. Otterness IG, Eckstein F (2007) Women have thinner cartilage and smaller joint surfaces than men after adjustment for body height and weight. Osteoarthritis Cartilage 15: 666672.

34. Faber SC, Eckstein F, Lukasz S, Muhlbauer R, Hohe J, et al. (2001) Gender differences in knee joint cartilage thickness, volume and articular surface areas: assessment with quantitative three-dimensional MR imaging. Skeletal Radiol 30: 144-150.

35. Ding C, Cicuttini F, Scott F, Glisson M, Jones G (2003) Sex differences in knee cartilage volume in adults: role of body and bone size, age and physical activity. Rheumatology (Oxford) 42: 1317-1323.

36. Cinotti G, Sessa P, Ragusa G, Ripani FR, Postacchini R, et al. (2013) Influence of cartilage and menisci on the sagittal slope of the tibial plateaus. Clin Anat 26: 883-892.

37. Balcarek $P$, Jung $K$, Ammon J, Walde TA, Frosch S, et al. (2010) Anatomy of lateral patellar instability: trochlear dysplasia and tibial tubercle-trochlear groove distance is more pronounced in women who dislocate the patella. Am J Sports Med 38: 2320-2327.

38. Fithian DC, Paxton EW, Stone ML, Silva P, Davis DK, et al. (2004) Epidemiology and natural history of acute patellar dislocation. Am J Sports Med 32: 1114-1121.

39. Jenny J, Honecker S, Chammai Y (2017) Radiographic measurement of the posterior femoral offset is not precise. Knee surgery, sports traumatology, arthroscopy: official journal of the ESSKA 25: 2609-2615.

40. Cheng FB, Ji XF, Zheng WX, Lai Y, Cheng KL, et al. (2010) Use of anthropometric data from the medial tibial and femoral condyles to design unicondylar knee prostheses in the Chinese population. Knee Surg Sports Traumatol Arthrosc 18: 352-358.

41. Shang $P$, Zhang L, Hou Z, Bai X, Ye X, et al. (2014) Morphometric measurement of the patella on $3 D$ model reconstructed from CT scan images for the southern Chinese population. Chin Med J 127: 96-101.

42. Wang J, Yue B, Wang Y, Yan M, Zeng Y (2012) The 3D analysis of the sagittal curvature of the femoral trochlea in the Chinese population. Knee Surg Sports Traumatol Arthrosc 20: 957-963.

43. Yan M, Wang J, Wang Y, Zhang J, Yue B, et al. (2014) Gender-based differences in the dimensions of the femoral trochlea and condyles in the Chinese population: correlation to the risk of femoral component overhang. Knee 21: 252-256. 
44. Ristic V, Maljanovic MC, Pericin B, Harhaji V, Milankov M (2014) The relationship between posterior tibial slope and anterior cruciate ligament injury. Med Pregl 67: 216-221.

45. Zhang Y, Wang J, Xiao J, Zhao L, Li ZH, et al. (2014) Measurement and comparison of tibial posterior slope angle in different methods based on three-dimensional reconstruction. Knee 21: 694-698.

46. Moghtadaei M, Moghimi J, Shahhoseini G (2015) Computed Tomographic measurement of distal femor rotation in Iranian population. Med J Islam Repub Iran 29: 169.

47. Raju S, Chinnakkannu K, Sunderayan R, Puttaswamy MK (2015) Rotational landmarks of the distal femur in Indian population: A MRI-based study. J Orthop Surg Res 10: 186.

48. Dickschas J, Harrer J, Bayer T, Schwitulla J, Strecker W (2016) Correlation of the tibial tuberosity-trochlear groove distance with the Q-angle. Knee Surg Sports Traumatol Arthrosc 24: 915-920.

49. Pandit S, Frampton C, Stoddart J, Lynskey T (2011) Magnetic resonance imaging assessment of tibial tuberosity-trochlear groove distance: normal values for males and females. Int Orthop 35: 1799-1803.

50. Skelley N, Friedman M, McGinnis M, Smith C, Hillen T, et al. (2015) Inter- and intraobserver reliability in the MRI measurement of the tibial tubercle-trochlear groove distance and trochlea dysplasia. Am J Sports Med 43: 873-878.

51. Han H, Oh S, Chang CB, Kang SB (2016) Anthropometric difference of the knee on MRI according to gender and age groups. Surg Radiol Anat 38: 203-211.

52. van Diek FM, Wolf MR, Murawski CD, van Eck CF, Fu FH (2014) Knee morphology and risk factors for developing an anterior cruciate ligament rupture: an MRI comparison between ACL-ruptured and non-injured knees. Knee Surg Sports Traumatol Arthrosc 22: 987-994.

53. Alemparte J, Ekdahl M, Burnier L, Hernandez R, Cardemil A, et al. (2007) Patellofemoral evaluation with radiographs and computed tomography scans in 60 knees of asymptomatic subjects. Arthroscopy 23: 170-177.

54. Nishimura K, Tanabe T, Kimura M, Harasawa A, Karita K, et al. (2005) Measurement of articular cartilage volumes in the normal knee by magnetic resonance imaging: can cartilage volumes be estimated from physical characteristics? J Orthop Sci 10: 246-252.

55. Antony B, Venn A, Cicuttini F, March L, Blizzard L, et al. (2015) Body composition, hormonal and inflammatory factors are associated with tibial cartilage volume in young adults and contribute to the sex difference in cartilage volume. Arthritis Care Res (Hoboken).

56. Berry PA, Wluka AE, Davies-Tuck M, Wang Y, Strauss BJ, et al. (2011) Sex differences in the relationship between bone mineral density and tibial cartilage volume. Rheumatology (Oxford) 50: 563-568.

57. Caglar E, Sahin G, Ogur T, Aktas E (2014) Quantitative evaluation of hyaline articular cartilage T2 maps of knee and determine the relationship of cartilage T2 values with age, gender, articular changes. Eur Rev Med Pharmacol Sci 18: 3386-3393.

58. Joseph GB, McCulloch CE, Nevitt MC, Heilmeier U, Nardo $\mathrm{L}$, et al. (2015) A reference database of cartilage $3 \mathrm{~T} \mathrm{MRI}$ $\mathrm{T} 2$ values in knees without diagnostic evidence of cartilage degeneration: data from the osteoarthritis initiative. Osteoarthr Cartil 23: 897-905.

59. Mosher TJ, Collins CM, Smith HE, Moser LE, Sivarajah RT, et al. (2004) Effect of gender on in vivo cartilage magnetic resonance imaging T2 mapping. J Magn Reson Imaging 19: 323-328.

60. Eckstein F, Faber S, Mühlbauer R, Hohe J, Englmeier KH, et al. (2002) Functional adaptation of human joints to mechanical stimuli. Osteoarthritis Cartilage 10: 44-50.

61. Draper CE, Besier TF, Gold GE, Fredericson M, Fiene A, et al. (2006) Is cartilage thickness different in young subjects with and without patellofemoral pain? Osteoarthr Cartil 14: 931-937.

62. Eckstein F, Yang M, Guermazi A, Roemer FW, Hudelmaier $M$, et al. (2010) Reference values and Z-scores for subregional femorotibial cartilage thickness--results from a large population-based sample (Framingham) and comparison with the non-exposed Osteoarthritis Initiative reference cohort. Osteoarthr Cartil 18: 1275-1283.

63. Cicuttini FM, Wluka AE, Wang Y, Davis SR, Hankin J, et al. (2002) Compartment differences in knee cartilage volume in healthy adults. J Rheumatol 29: 554-556.

64. Beattie KA, Duryea J, Pui M, O'Neill J, Boulos P, et al. (2008) Minimum joint space width and tibial cartilage morphology in the knees of healthy individuals: a cross-sectional study. BMC Musculoskelet Disord 9: 119.

65. Eckstein F, Siedek V, Glaser C, Al-Ali D, En glmeier KH, et al. (2004) Correlation and sex differences between ankle and knee cartilage morphology determined by quantitative magnetic resonance imaging. Ann Rheum Dis 63: 14901495.

66. Berry PA, Teichtahl AJ, Galevska-Dimitrovska A, Hanna FS, Wluka AE, et al. (2008) Vastus medialis cross-sectional area is positively associated with patella cartilage and bone volumes in a pain-free community-based population. Arthritis Res Ther 10: R143.

67. Li K, Langdale E, Tashman S, Harner C, Zhang X (2012) Gender and condylar differences in distal femur morphometry clarified by automated computer analyses. J Orthop Res 30: 686-692.

68. Barnes CL, Iwaki H, Minoda Y, Green JM, Obert RM (2010) Analysis of sex and race and the size and shape of the distal femur using virtual surgery and archived computed tomography images. J Surg Orthop Adv 19: 200-208.

69. Park JS, Nam DC, Kim DH, Kim HK, Hwang SC (2012) Measurement of Knee Morphometrics Using MRI: A Comparative Study between ACL-Injured and Non-Injured Knees. Knee Surg Relat Res 24: 180-185.

70. Fehring TK, Odum SM, Hughes J, Springer BD, Beaver WB Jr (2009) Differences between the sexes in the anatomy of the anterior condyle of the knee. J Bone Joint Surg Am 91: 2335-2341.

71. Yue B, Wang J, Wang Y, Yan M, Zhang J, et al. (2015) The intercondylar notch ceiling: an accurate reference for distal femoral resection in total knee arthroplasty for severely degenerated varus knees. Knee Surg Sports Traumatol Arthrosc 25: 2818-2824.

72. Rosenstein AD, Veazey B, Shephard D, Xu KT (2008) Gender differences in the distal femur dimensions and variation patterns in relation to TKA component sizing. Orthopedics 31: 652 .

73. Li P, Tsai TY, Li JS, Wang S, Zhang Y, et al. (2014) Gender analysis of the anterior femoral condyle geometry of the knee. Knee 21: 529-533.

74. Murshed KA, Cicekcibasi AE, Karabacakoglu A, Seker M, 
Ziylan T (2005) Distal femur morphometry: a gender and bilateral comparative study using magnetic resonance imaging. Surg Radiol Anat 27: 108-112.

75. Vrooijink SH, Wolters F, Van Eck CF, Fu FH (2011) Measurements of knee morphometrics using MRI and arthroscopy: a comparative study between ACL-injured and non-injured subjects. Knee Surg Sports Traumatol Arthrosc 9: $12-16$

76. Anderson AF, Dome DC, Gautam S, Awh MH, Rennirt GW (2001) Correlation of anthropometric measurements, strength, anterior cruciate ligament size, and intercondylar notch characteristics to sex differences in anterior cruciate ligament tear rates. Am J Sports Med 29: 58-66.

77. van Eck CF, Kopf S, van Dijk CN, Fu FH, Tashman S (2011) Comparison of 3-dimensional notch volume between subjects with and subjects without anterior cruciate ligament rupture. Arthroscopy 27: 1235-1241.

78. van Eck CF, Martins CA, Lorenz SG, Fu FH, Smolinski $P$ (2010) Assessment of correlation between knee notch width index and the three-dimensional notch volume. Knee Surg Sports Traumatol Arthrosc 18: 1239-1244.

79. Charlton WP, St John TA, Ciccotti MG, Harrison N, Schweitzer M (2002) Differences in femoral notch anatomy between men and women: a magnetic resonance imaging study. Am J Sports Med 30: 329-333.

80. Dienst M, Schneider G, Altmeyer K, Voelkering K, Georg T, et al. (2007) Correlation of intercondylar notch cross sections to the ACL size: a high resolution MR tomographic in vivo analysis. Arch Orthop Trauma Surg 127: 253-260.

81. Estes K, Cheruvu B, Lawless M, Laughlin R, Goswami T, et al. (2015) Risk assessment for anterior cruciate ligament injury. Arch Orthop Trauma Surg 135: 1437-1443.
82. Biedert RM, Bachmann M (2009) Anterior-posterior trochlear measurements of normal and dysplastic trochlea by axial magnetic resonance imaging. Knee Surg Sports Traumatol Arthrosc 17: 1225-1230.

83. Hasler RM, Gal I, Biedert RM (2014) Landmarks of the normal adult human trochlea based on axial MRI measurements: a cross-sectional study. Knee Surg Sports Traumatol Arthrosc 22: 2372-2376.

84. Kamath AF, Slattery TR, Levack AE, Wu CH, Kneeland JB, et al. (2013) Trochlear inclination angles in normal and dysplastic knees. J Arthroplasty 28: 214-219.

85. Lee UY, Kim IB, Kwak DS (2015) Sex determination using discriminant analysis of upper and lower extremity bones: New approach using the volume and surface area of digital model. Forensic Sci Int 253: 135.

86. Bellemans J, Carpentier K, Vandenneucker H, Vanlauwe J, Victor J (2010) The John Insall Award: Both morphotype and gender influence the shape of the knee in patients undergoing TKA. Clin Orthop Relat Res 468: 29-36.

87. Yoo JH, Yi SR, Kim JH (2007) The geometry of patella and patellar tendon measured on knee MRI. Surg Radiol Anat 29: 623-628.

88. Kucukdurmaz F, Tuncay I, Elmadag M, Tuncer N (2014) Morphometry of the medial tibial plateau in Turkish knees: correlation to the current tibial components of unicompartmental knee arthroplasty. Acta Orthop Traumatol Turc 48: 147-151.

89. Stone KR, Freyer A, Turek T, Walgenbach AW, Wadhwa S, et al. (2007) Meniscal sizing based on gender, height, and weight. Arthroscopy 23: 503-508. 